\title{
PRINCIPAL FIBRATIONS
}

\author{
BY \\ JEAN-PIERRE MEYER(1)
}

1. Introduction. As is well known, a fibration with an Eilenberg-MacLane space of type $(\pi, n), n>1$, as fiber and simply-connected base is equivalent to one induced from a path-space fibration by a map of the base into an EilenbergMacLane space of type $(\pi, n+1)$. For the purposes of this paper, and by analogy with the classification theory of fiber-bundles, we call such a fibration principal. The problem considered here is the following: under what conditions is a fibration with a loop-space as fiber equivalent to a principal one? In view of the BarrattGugenheim-Moore classification theory of semi-simplicial fibrations (Amer. J. Math. 81 (1959)), this problem is equivalent, heuristically at any rate, to stating conditions under which the group of the fibration (the automorphism group of the fiber) can be reduced to the fiber itself. Since we prefer to remain in the purely topological context, where the implications of the semi-simplicial theory are not clear, we follow another approach and obtain a general answer to the question (§ 3). As an application, we verify a conjecture left open in [3].

In $\$ 5$, we introduce $H$-fibrations which stand in the same relation to principal fibrations as $H$-spaces do to loop-spaces. Various properties of $H$-fibrations are obtained which generalize the well-known theorems concerning the vanishing of Whitehead products and the primitivity of the $k$-invariants of $H$-spaces.

It is almost unnecessary, by now, to remark that most of the definitions, theorems, and proofs which follow can be dualized à la Eckmann-Hilton to yield theorems concerning principal cofibrations.

2. Preliminaries. We recall, in this section, some definitions due to EckmannHilton [1], and prove some elementary lemmas.

All spaces are assumed to have the homotopy type of a $C W$-complex [2], to have base-points; all maps (homotopies) are assumed to preserve (keep fixed) basepoints. Base-points and mappings into base-points will often be denoted by $*$.

The standard Serre path-space fibration will be denoted by $\pi: E X \rightarrow X$, $\pi(\tilde{\omega})=\tilde{\omega}(1)$, its fiber by $\Omega X ; \pi(A, B)$ denotes the set of homotopy classes of maps $A \rightarrow B, \pi_{1}(\alpha, B)$ the set of homotopy classes of commutative diagrams

Received by the editors May 24, 1961 and, in revised form, June 5, 1962.

(1) This work was partially supported by the Army Research Office under Contract DA-36034-ORD-3299RD. 


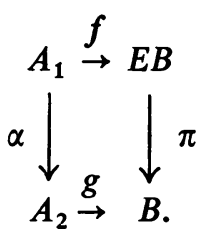

Such a diagram, with $\alpha$ and $\pi$ fixed, will sometimes be denoted in abbreviated fashion by $(f, g)$ and a homotopy between two such diagrams by $\left(f_{t}, g_{t}\right)$.

The sets $\pi(A, B), \pi(A, \Omega B)$ may also be considered, respectively, as the sets of homotopy classes of commutative diagrams

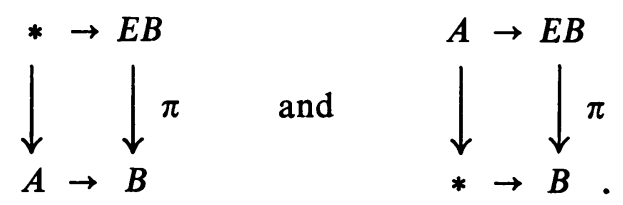

In view of this interpretation we may define certain mappings. First, $J: \pi\left(A_{1} ; \Omega B\right) \rightarrow \pi_{1}(\alpha ; B)$, the image of

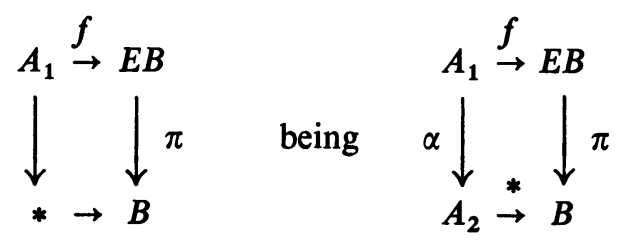

Secondly, if

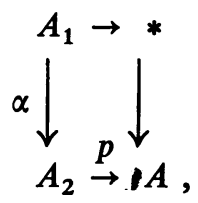

then $p^{*}: \pi(A, B) \rightarrow \pi_{1}(\alpha, B)$, the image of

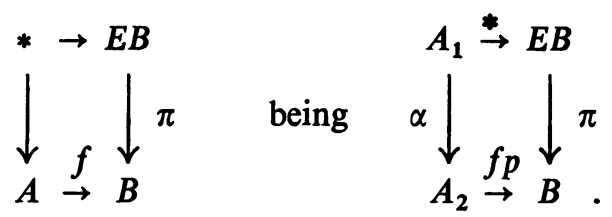

(2.1) LeMmA. If $\sigma: \Omega B \rightarrow \Omega B$ is the inverse map, $\sigma \tilde{\omega}(s)=\tilde{\omega}(1-s)$, then $J[\sigma]=\pi^{*}[1]$ in the diagram:

$$
\pi(\Omega B, \Omega B) \stackrel{J}{\rightarrow} \pi_{1}(j ; B) \stackrel{\pi^{*}}{\leftarrow} \pi(B, B)
$$

where $j: \Omega B \rightarrow E B$ is the inclusion. 
Proof. Since $[\sigma]$ is represented by $(j \sigma, *)$ and $[1]$ by $(*, 1)$ the elements $J[\sigma]$ and $\pi^{*}[1]$ are represented, in the diagram below, by $(j \sigma, *)$ and $(*, \pi)$, respectively:

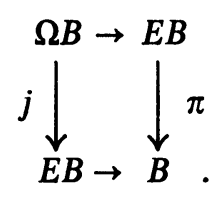

A homotopy between these two diagrams is given by $\left(f_{t}, g_{t}\right)$ where $f_{t}(\tilde{\omega})(s)=$ $\tilde{\omega}(1-s[1-t]), \tilde{\omega} \in \Omega B$ and $g_{t}(\tau)=\tau(t), \tau \in E B$.

(2.2) LemMa. If $f_{t}: A \rightarrow E B$ is a null-homotopy of if $\left(f_{0}=*, f_{1}=\right.$ if) with $f: A \rightarrow \Omega B$ and $f^{\prime}: A \rightarrow \Omega B$ is defined by $f^{\prime}(a)(s)=\pi f_{1-s}(a)$, then $f^{\prime} \simeq \sigma f$, or equivalently $\left[f^{\prime}\right]=-[f]$.

Proof. For each $a \in A, f_{t}$ defines a map of the square $0 \leqq s \leqq 1,0 \leqq t \leqq 1$ which maps the sides $s=0$ and $t=0$ to the base-point $*$, which maps $t=1,0 \leqq s \leqq 1$ according to $f(a)$ and $s=1,0 \leqq t \leqq 1$ according to $\sigma f^{\prime}(a)$ :

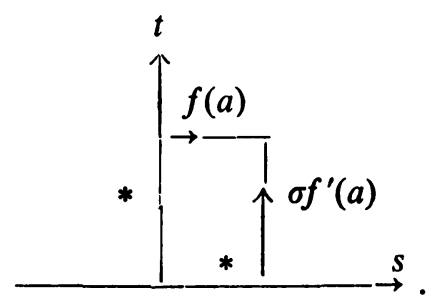

Thus it is easily seen that $f \simeq \sigma f^{\prime}$ or $f^{\prime} \simeq \sigma f$.

(2.3) LEMMA. If

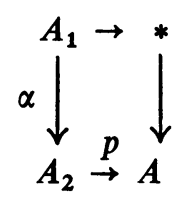

and $\bar{p}: A_{2} \cup_{\alpha} C A_{1} \rightarrow A$ is defined by $\bar{p}\left|A_{2}=p, \bar{p}\right| C A_{1}=*$, then there is a oneone mapping $\theta$ such that the following diagram is commutative:

$$
\begin{gathered}
\pi_{1}(\alpha, B) \stackrel{\theta}{\rightarrow} \pi\left(A_{2} \cup_{\alpha} C A_{1} ; B\right) \\
\pi(A, B)
\end{gathered}
$$

The proof is straight forward. 


\section{The main theorem.}

(3.1) Definition. A fibration $p: E \rightarrow B$ is principal if there exists a "classifying space" $A$ and a "classifying map" $k: B \rightarrow A$ such that $p$ is induced from $\pi: E A \rightarrow A$ by $k$.

If $p$ is principal, the fiber is $\Omega A$ and there is an operation $\mu: \Omega A \times E \rightarrow E$ such that the following diagram is commutative:

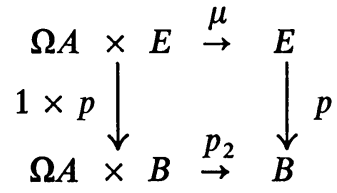

where $p_{2}$ is the projection onto the second factor. Such fibrations possess most of the useful properties of principal fiber-bundles: they have cross-sections if and only if they are equivalent to products, the homology of the fiber operates on the homology spectral sequence, etc.

In particular, the following lemma holds:

(3.2) Lemma. (a) $f: X \rightarrow B$ can be lifted to a map $X \rightarrow E$ if and only if $k f \simeq *$.

(b) If $f, g: X \rightarrow E$ are such that $p f \simeq p g$, then there is a map $d: X \rightarrow \Omega A$ such that $g \simeq \mu(d \times f) \Delta$, where $\Delta: X \rightarrow X \times X$ is the diagonal map.

The proof is elementary; see, for example [4]. The map $\mu(d \times f) \Delta$ will be denoted by $f^{d}$.

(3.3) Definition. The fibrations $(E, B, F, p)$ and $\left(E^{\prime}, B, F^{\prime}, p^{\prime}\right)$ are equivalent if there exists a map $q: E \rightarrow E^{\prime}$ such that:

(a) $p^{\prime} q=p$.

(b) $q$ is a homotopy equivalence.

The map $q \mid F$ is the induced fiber equivalence; it is a homotopy equivalence, as is easily seen from the homotopy ladder of $q$, and the 5-lemma.

(3.4) Theorem. Let $F \rightarrow{ }^{i} E \rightarrow{ }^{p} B$ be a fibration and $l: F \rightarrow \Omega A$ a homotopyequivalence. Then $p$ is equivalent to a principal fibration with induced fiberequivalence in $[l]$ if and only if $J[\sigma l] \in$ image $p^{*}$.

Proof $\left({ }^{2}\right)$. Assume there is $k: B \rightarrow A$ with $E^{\prime}$ induced from $E A$ by $k$ and an equivalence $q: E \rightarrow E^{\prime}$ inducing $l^{\prime} \simeq l$. Then we have

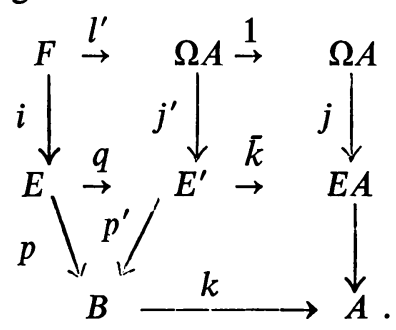

(2) This is a simplified version of the original proof, following a suggestion of the referee. 
Thus we have the commutative diagram

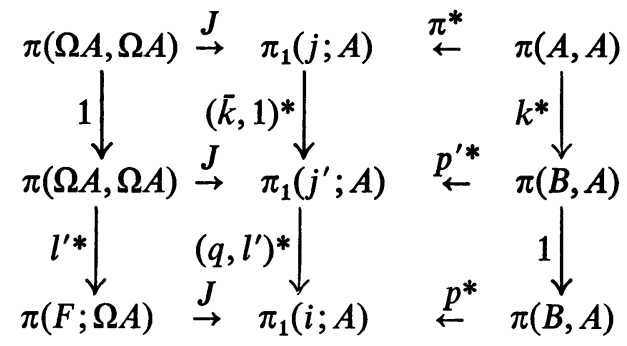

and it follows, using Lemma (2.1) that $J l^{*}[\sigma]=p^{*}[k]$; but $l^{\prime *}[\sigma]=[\sigma l]$ and one implication is proved.

Conversely, suppose there is $k: B \rightarrow A$ such that $J[\sigma l]=p^{*}[k]$. Then $p^{*}[k]$, $J[\sigma l]$ being represented, respectively, by $(*, k p)$ and $(j \sigma l, *)$ there is a homotopy

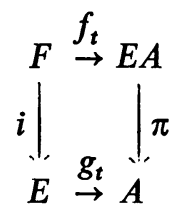

with $f_{0}=^{*}, g_{0}=k p, f_{1}=j \sigma l, g_{1}=^{*}$. Define $k^{\prime}: E \rightarrow E A$ by $k^{\prime}(e)(t)=g_{1-t}(e)$. Then $\pi k^{\prime}(e)=k^{\prime}(e)(1)=g_{0}(e)=k p(e)$, and if $f \in F, k^{\prime} i(f)(t)=g_{1-t}($ if $)=\pi f_{1-t}(f)$. Thus, if $l^{\prime}=k^{\prime} \mid F: F \rightarrow \Omega A$, it follows from Lemma (2.2) that $l^{\prime} \simeq \sigma \sigma l=l$. If we now define $q: E \rightarrow E^{\prime}$ by $q(e)=\left(p(e), k^{\prime}(e)\right)$, it follows from the homotopy ladder of $q$ and the 5-lemma ( since $l^{\prime}$ is an equivalence) that $q$ is an equivalence.

(3.5) COROLLARY. Under the hypotheses of (3.4), p is equivalent to a principal fibration with induced fiber equivalence in $[l]$ if and only if $\theta J[\sigma l] \in$ image $\bar{p}^{*}$.

This is a consequence of Lemma (2.3).

4. An application. In this section, we apply (3.5) to verify a conjecture discussed in (4.2) of [3].

(4.1) LeMma. Let $\alpha: A_{1} \rightarrow A_{2}$ be a map such that $\alpha_{*}: H_{i}\left(A_{1}\right) \rightarrow H_{i}\left(A_{2}\right)$ is an isomorphism for $i<N$ and an epimorphism for $i=N$. Then if $\pi_{i}(B)=0$, $i \geqq N(i \geqq N+1), \alpha^{*}: \pi\left(A_{2}, B\right) \rightarrow \pi\left(A_{1}, B\right)$ is a surjection (injection).

Proof. Let $\bar{\alpha}$ be an inclusion (obtained, for example, via the mapping cylinder) equivalent to $\alpha$ :

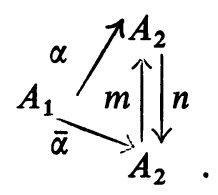

The maps $m, n$ form a homotopy-equivalence. Then $m^{*}: \pi\left(A_{2}, B\right) \rightarrow \pi\left(A_{3}, B\right)$ and 
$n^{*}: \pi\left(A_{3}, B\right) \rightarrow \pi\left(A_{2}, B\right)$ are inverses of each other and one need only study $\bar{\alpha}^{*}$. This can be done by obstruction theory. The hypothesis on $\alpha_{*}$ implies that $H_{i}\left(A_{3}, A_{1}\right)=0, i \leqq N$. Hence, if $[a] \in \pi\left(A_{1}, B\right)$, the obstructions to extending $a$ to $A_{3}$ belong to $H^{j+1}\left(A_{3}, A_{1} ; \pi_{j}(B)\right)$, which vanishes for all $j$ by the universal coefficient theorem, if $\pi_{j}(B)=0, i \geqq N$.

Similarly, if $a_{0}, a_{1}: A_{3} \rightarrow B$ are extensions of $a$, the obstructions to a homotopy between them belong to $H^{j}\left(A_{3}, A_{1} ; \pi_{j}(B)\right)$, which vanishes for all $j$ if $\pi_{j}(B)=0$, $j \geqq N+1$.

(4.2) THEOREM. Let $F \rightarrow^{i} E \rightarrow{ }^{p} B$ be a fibration, satisfying the following conditions:

(a) $\pi_{i}(B)=0, i<p$;

(b) $\pi_{i}(F)=0, i<q, i>p+q-2,2 \leqq p \leqq q$.

Then $p$ is equivalent to a principal fibration $\left({ }^{3}\right)$.

Proof. As $p \leqq q, p+q-2 \leqq 2 q-2$ and $F$ has the homotopy type of a loopspace $\Omega A$. Let $l: F \rightarrow \Omega A$ be an equivalence. By a classical result of Serre [5], $p_{*}: H_{i}(E, F) \rightarrow H_{i}(B)$ is an isomorphism for $i \leqq p+q-1$ and an epimorphism for $i=p+q$; hence $\bar{p}_{*}: H_{i}(E \cup C F) \rightarrow H_{i}(B)$ has the same property.

On the other hand, $\pi_{i}(A)=0, i \geqq p+q$; so, by $(4.1), \bar{p}^{*}: \pi(B, A) \rightarrow \pi(E \cup C F, A)$ is an isomorphism. The theorem now follows from (3.5).

(4.3) Corollary. If $X_{i}$ denotes the ith space in a Postnikov system of a $(p-1)$ connected space $X, p \geqq 2$, then the composite fibration $X_{p+q-2} \rightarrow X_{q-1}$ is equivalent to a principal fibration.

This is the conjecture referred to above.

5. $H$-fibrations and Whitehead products. In attempting to generalize Theorem (4.2) it seems natural to introduce a concept intermediate between that of an arbitrary fibration and of a principal fibration, and which stands in the same relation to principal fibrations as the notion of $H$-space does to that of loop-space.

(5.1) Definition. The fibration $F \rightarrow E \rightarrow^{p} B$ is an $H$-fibration if there exists a map (the operation) $\mu: F \times E \rightarrow E$ and a homotopy $H_{t}: F \vee E \rightarrow E$ satisfying the conditions:

(a) the diagram

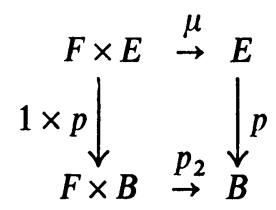

is commutative.

(3) The present author stated this theorem as a conjecture in [3] and remarked in a footnote, added in proof, that he had found a proof; proofs have also been given by T. Ganea [6], P. J. Hilton [7], and Y. Nomura [8]. 
(b) $H_{0}=\mu j, H_{1}=\nabla(i \vee 1), p H_{t}(F \vee F)=*$, where $j$ is the inclusion $F \vee E \rightarrow F \times E, \nabla$ the folding map $E \vee E \rightarrow E$.

It is clear that $\mu \mid F \times F$ and $H_{t} \mid F \vee F$ define an $H$-space structure on $F$. In this section, we obtain generalizations of the well-known theorems concerning the vanishing of Whitehead products and the primitivity of the $k$-invariants of an $H$-space.

(5.2) Proposition. If $p$ is an $H$-fibration, $\alpha \in \pi_{p}(F), \beta \in \pi_{q}(E)$, then $\left[i_{*}(\alpha), \beta\right]=0$.

Proof. Let $f: S^{p} \rightarrow F, g: S^{q} \rightarrow E$ represent $\alpha$ and $\beta$. Then, in the following diagram, the squares are homotopy-commutative:

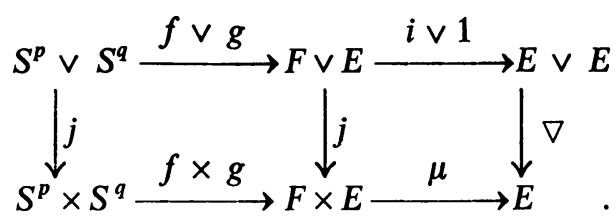

Thus the map $S^{p} \vee S^{q} \rightarrow E$ is extendable to $S^{p} \times S^{q}$ and so $\left[i_{*}(\alpha), \beta\right]=0$.

(5.3) Definition. Let $K$ be an $H$-space with multiplication $m, p$ an $H$-fibration with operation $\mu$, and $k: E \rightarrow K$. Then $k$ is primitive if the following diagram is homotopy-commutative:

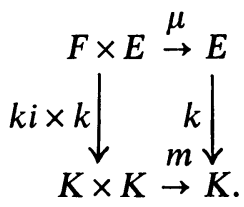

(5.4) TheOREM. Let $p, k, K$ be as in (5.3) and $t: E^{\prime} \rightarrow E$ the fibration induced by $k$ from $E K \rightarrow K, p^{\prime}=p t$. Then $p^{\prime}$ is an H-fibration if $k$ is primitive.

Proof. The proof is a straightforward generalization of that for the case $B=*$ (see, for example [1, p. 294]). Let $i^{\prime}: F^{\prime} \rightarrow E^{\prime}, j^{\prime}: F^{\prime} \vee E^{\prime} \rightarrow F^{\prime} \times E^{\prime}, j: F \vee E$ $\rightarrow F \times E$ be the inclusions, $s=t \mid F^{\prime}: F^{\prime} \rightarrow F$.

Consider $\mu(s \times t): F^{\prime} \times E^{\prime} \rightarrow E$. Since $k t \simeq *$,

$$
k \mu(s \times t) \simeq m(k i \times k)(s \times t)=m(k i s \times k t)=m\left(k t i^{\prime} \times k t\right) \simeq *,
$$

and there is, by (3.2a), $\lambda: F^{\prime} \times E^{\prime} \rightarrow E^{\prime}$ such that $t \lambda=\mu(s \times t)$. Now $t \lambda j^{\prime} \simeq t \nabla\left(i^{\prime} \vee 1\right)$ and so, by (3.2b), there is a map $d: F^{\prime} \vee E^{\prime} \rightarrow \Omega K$ such that $\nabla\left(i^{\prime} \vee 1\right) \simeq\left(\lambda j^{\prime}\right)^{d}$.

As $\Omega K$ is an $H$-space (with multiplication $\bar{m}$ ), the map

$$
\delta=\bar{m}\left\{\left(d \mid F^{\prime}\right) \times\left(d \mid E^{\prime}\right)\right\}: F^{\prime} \times E^{\prime} \rightarrow \Omega K
$$

has the property $\delta j^{\prime} \simeq d$, so 


$$
\nabla\left(i^{\prime} \vee i\right) \simeq\left(\lambda j^{\prime}\right)^{\delta j^{\prime}}=\left(\lambda^{\delta}\right) j^{\prime}
$$

Let $\mu^{\prime}=\lambda^{\delta}$. Then conditions (a) and (b) are satisfied.

We may apply (5.4) to study the successive fibrations in a Moore-Postnikov system of a fibration $f: X \rightarrow B$. Let the fibrations be denoted by $p_{i}: X_{i} \rightarrow X_{i-1}$, $X_{0}=B, p_{i}^{-1}(*)=K\left(\pi_{i}(F), i\right)$, and let $f_{i}$ denote the composition

$$
p_{1} p_{2} \cdots p_{i}: X_{i} \rightarrow B, F_{i}=f_{i}^{-1}(*) \text {. }
$$

(5.5) Corollary. Let $B$ be $(p-1)$-connected and $F$ be $(q-1)$-connected $(p \leqq q)$. Then $f_{i}: X_{i} \rightarrow B$ is an H-fibration, if $i \leqq p+q-2$.

Proof. The proof proceeds by induction on $i$ and follows immediately from the observation that the first cross-term in $H^{i+1}\left(F_{i-1} \times X_{i-1} ; \pi_{i}\right)$ occurs when $i=p+q-1$, so that, if $i \leqq p+q-2$,

$$
\mu_{i-1} * k_{i+1} * b^{i+1}=\pi_{1} * i * k_{i+1} * b^{i+1}+\pi_{2} * k_{i+1} * b^{i+1},
$$

where $\pi_{1}, \pi_{2}$ are the projections, $k_{i+1}: X_{i-1} \rightarrow K\left(\pi_{i}, i+1\right)$ the classifying map and $b^{i+1}$ the basic class. Hence $k_{i+1}$ is primitive if $i \leqq p+q-2$ and the conclusion follows from (5.4).

The above corollary is, of course, a weaker form of (4.2). It is of interest because it indicates how the notion of $H$-fibration can be studied via Moore-Postnikov systems. The first "obstruction to primitivity," as was seen in the proof of (5.5), is a cross-term of $H^{p+q}\left(F_{p+q-2} \times X_{p+q-2} ; \pi_{p+q-1}\right)$, and therefore lies in a group isomorphic to $\operatorname{Hom}\left(H_{q}\left(F_{p+q-2}\right) \otimes H_{p}\left(X_{p+q-2}\right) ; \pi_{p+q-1}\right)$.

If $p<q$, so that $p+q-1<2 q-1$ and $F_{p+q-1}$ is an $H$-space, this group is isomorphic to $\operatorname{Hom}\left(\pi_{q}(F) \otimes \pi_{p}(X), \pi_{p+q-1}(F)\right)$. Finally, if $\partial: \pi_{p+q}(B) \rightarrow \pi_{p+q-1}(F)$ vanishes, so that $i_{*}: \pi_{p+q-1}(F) \rightarrow \pi_{p+q-1}(X)$ is a monomorphism, the obstruction to primitivity can be identified, by a method completely analogous to that of [3], to the Whitehead product $\pi_{q}(F) \otimes \pi_{p}(X) \rightarrow \pi_{p+q-1}(F)$ defined by

$$
a \otimes b \rightarrow i_{*}^{-1}\left[i_{*}(a), b\right] \text {. }
$$

(5.6) THEOREM. If $X_{i}$ denotes the ith space in a Postnikov system of a $(p-1)$ connected space $X, p \geqq 2$, then $X_{p+q-1} \rightarrow X_{q-1}(p<q)$ is an H-fibration if and only if the Whitehead product $\pi_{p}(X) \otimes \pi_{q}(X) \rightarrow \pi_{p+q-1}(X)$ vanishes.

Proof. Let $B=X_{q-1}$. Then $X_{p+q-2} \rightarrow B$ is an $H$-fibration by (5.5), and $\pi_{p+q}(B)=0$. Hence the above argument applies and the first implication follows from (5.4) and the fact that, if $F$ denotes the fiber of $X_{p+q-1} \rightarrow X_{q-1}$, then $i_{*}: \pi_{j}(F) \rightarrow \pi_{j}\left(X_{p+q-1}\right)$ is an isomorphism for $q \leqq j \leqq p+q-1$. The other implication is a consequence of (5.2).

In view of the fact that $H$-fibrations, as seen above, can be studied using their Postnikov systems by cohomological methods, it would be of interest to find general conditions under which an $H$-fibration is also a principal one. 


\section{REFERENCES}

1. P. J. Hilton, Homotopy theory and duality, mimeographed notes, Cornell University, 1959; cf. also, Groupes d'homotopie et dualité, C. R. Acad. Sci. Paris 246 (1958), pp. 2444, 2555, 2991.

2. J. W. Milnor, On spaces having the homotopy-type of a CW-complex, Trans. Amer. Math. Soc. 90 (1959), 272-280.

3. J.-P. Meyer, Whitehead products and Postnikov systems, Amer. J. Math. 82 (1960), 271-280.

4. F. P. Peterson and E. Thomas, $A$ note on non-stable cohomology operations, Bol. Soc. Mat. Mexicana 3 (1958), 13-18.

5. J.-P. Serre, Homologie singulière des espaces fibrés, Ann. of Math. (2) 54 (1951), 425-505.

6. T. Ganea, Fibrations and cocategory, Comment. Math. Helv. 35 (1961), 15-24.

7. P. J. Hilton, On excision and principal fibrations, Comment. Math. Helv. 35 (1961), 77-84.

8. Y. Nomura, A generalization of suspension theorems, Nagoya Math. J. 19 (1961), 159-167.

THE JOHNS HOPKINS UNIVERSITY,

Baltimore, MARYLAND 